\title{
MULTIVARIABLE CONTROL OF A TWIN LIFT HELICOPTER SYSTEM USING THE LQG/LTR DESIGN METHODOLOGY*
}

by

\author{
Armando A. Rodriguez and Michael Athans
}

Laboratory for Information and Decision Systems

Massachusetts institute of Technology

Cambridge, Massachusetts 02139

\begin{abstract}
This paper presents quidelines for developing a multivariable centralized automatic rlight control system (AFCS) for a twin lift helicopter system (TLHS). Singular value ideas are used to formulate performance and stability robustness specifications. A Linear-Quadratic-Gaussian with Loop Transfer Recovery (LQG/LTR) design is obtained and evaluated.
\end{abstract}

\section{Introduction}

\subsection{Background}

During recent years the development of heavy lift helicopters has leveled off. Users have found it difficult to justify the high costs associated with such a development, particularly when there are much more economical alternatives. Twin lift is such an alternative. It. a!lows the use of already existing helicopters to conauct missions which would otherwise de impossible.

This paper illustrates how the LQG/LTR design methodology [5] [6], coupled with singular value ideas [7] [8]. can be used to develop an automatic centralized feedback controller for a TLHS.

\subsection{Outline of Paper}

Because of the inherent coupling in TLHS's, single input-single output (SISO) design techniques can not easily be used to systematically develop a centralized AFCS. The paramount contribution of this paper is to present guidelines for designing a "real world" multi-input multi-output (MIMO) AFCS for a TLHS.

*This research was supported by the NASA Ames and Langley Research Centers under grant NASA/NAG-2-297.
The paper begins with a description of a model for the TLHS to be studied. A set of output variables are then selected. (These are the variables to be actually commanded by the pilot).

The cantrol problem is then formulated by discussing the structure of the final AFCS and giving performance and stability robustness specifications. Next, the LQG/LTR design methodology is described and used to obtain an AFCS. The AFCS is then evaluated by discussing the properties of the final loop. sensitivity. and closed loop transfer function matrix singular values. The characteristics of selected system transient responses to typical pilot reference commands are also discussed.

Finally, the flexibility of the methodology to meet prescribed piloz specifications is discussed.

2. Modeling a Twin Lift ielicopter System

\section{2.: Cescription of sustem}

For simplicity this paper only focusses an controlling the longitudinal dynamics of a TLHS near hovering trim. The configuration to be studied, called the longitudinal configuration, is shown in Fig. 1.

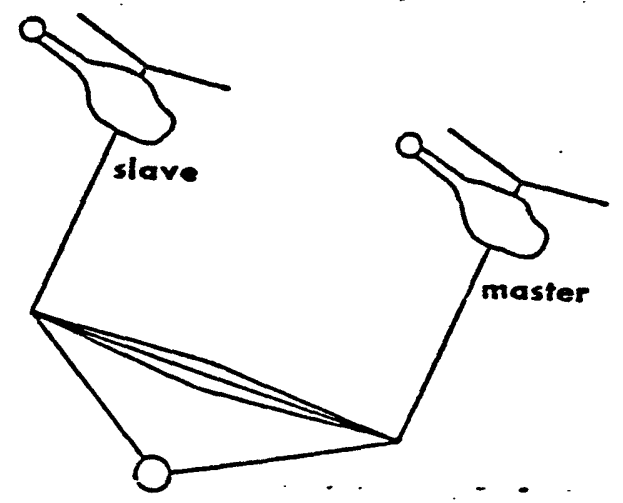

Fig. 1: Twin Lift Helicopter System (TLHS); Longitudinal Configuration. 
The longitudinal configuration consists of two helicopters, two tethers, a spreader bar, two load cables. and a payload. In studying this configuration it is assumed that the lateral dynamics of the helicopters and load-bar assembly decouple from the planar (longitudinal) dynamics of interest; i.e. the system motion is restricted to the vertical (longitudinal) plane and hence only vertical translation, horizontal translation, and pitching are "allowed".

The helicopters being modeled are Sikorsky UH-60A Blackhawks. They are assumed to be identical. The lead helicopter is referred to as the master helicopter, and the trail helicopter as the slave. Each tether is assumed to have a rixed length, $H$. The helicopter-tether attachment points are assumed to lie a fixed distance, $h$, below the center of gravities (c.g.'s) of the helicopters. Each tether is cornected to one end of the spreader bar which is assumed to be rigid and have a fixed length. $L$. The payload is suspended a fixed distance, $Z$, below the spreader bar cg. via two fixed length cables. For simplicity the teiners, as well as the load cabies, are assumed to have no compliance and to always be in tension

2.2 Variable derinitions and State Space Model

A seven degree of freedom linear model [I] is used to characterize the rigid body dynamics of the system. The key degrees of freedom are described as follows: three degrees of freedom per helicopter (vertical translation, horizontal transiation, and pitching), and one degree or rreedom for the load which is mudeled as a point mass (constrained perdular motion).

Since this study restricts the system motion to the longitudinal plane, only four controls (two per helicopter) are relevant. These are the collective controls and the cyclic controls. The collectives control the up/down motion of the helicopters. The cyclics control their fore/aft motion as well as their pitching motion. To fully exploit the symmetry of the "equal tether configuration" being addressed, the

following seven degrees of freedom [I] and four controls are def ined (Fig. 2):

Average Variables:

$\Sigma x \equiv\left(x_{m}+x_{s}\right) / 2$ : Average horiz. helic. coord.

$\Sigma z \equiv\left(z_{m}+z_{s}\right) / 2$ : Average vert. helic. coord.

$\Sigma \theta \equiv\left(\theta_{\mathrm{m}}+\theta_{\mathrm{s}}\right) / 2$ : Average helic. pitch attitude

$\Sigma \beta_{l C} \equiv\left(\beta_{l c m}+\beta_{l c s}\right) / 2$ : Average cyclic control
$\Sigma \theta_{c} \equiv\left(\theta_{c m}+\theta_{c S}\right) / 2$ : Average collective control (5) Difference Variables:

$\Delta x \equiv x_{m}-x_{s}$ : Horizontal separation

$\Delta z \equiv z_{m}-z_{s}$ : Vertical separation

$\Delta \theta \equiv \theta_{\mathrm{m}}-\theta_{\mathrm{s}}$ : Differential pitch attitude

$\Delta \beta_{\text {lc }} \equiv \beta_{\text {lcm }}-\beta_{\text {lcs }}$ : Differential cyclic control

$\Delta \theta_{c} \equiv \theta_{\mathrm{cm}}-\theta_{c s}$ : Dirferential collective control (10) where $\left|\beta_{l d}\right|<15$ degrees and $\left|\theta_{c}\right|<10$ degrees

for each helicopter.

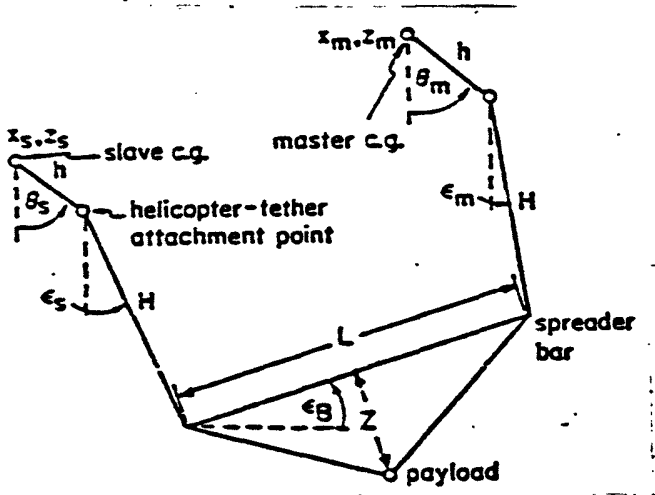

Fig. 2: Model for TLHS longitudinal dynamics.

The variables defined above are used to derive the equations which govern the planar motion of the TLHS. In doing so it is assumed that the trim condition is one in which the helicopters are near hover with the tethers vertical and the spreader bar horizontal. It is also assumed that the helicopter c.g's lie on the main rotor driveshafts. This assumption icrces the helicopters to have no nominal pitch for the trim condition described above. With these assumptions a linear state space model is constructed having the following form:

$\dot{x}_{p}=A_{p} \underline{x}_{p}+B_{p} u_{p}: \quad x_{p} \in R^{12} . u_{p} \in R^{4}$

$x_{p} \equiv\left[\Sigma \dot{z}\|\Delta x \Delta \theta \Delta \dot{x} \Delta \dot{\theta}\| \Sigma \theta \Delta z \Sigma E \sum \dot{x} \Sigma \dot{\theta} \Delta \dot{z} \sum \dot{\varepsilon}\right]^{T}$ (12)

$u_{p} \equiv\left[\Sigma \theta_{c}\left\|\Delta \beta_{\text {lc }}\right\| \Delta \theta_{c} \Sigma \beta_{\text {lc }}\right]^{\top}$

$A_{p}=\operatorname{diag}\left(A_{p 1} \cdot A_{p 2} \cdot A_{p 3}\right)$

$B_{p} \equiv \operatorname{diag}\left(B_{p 1}, B_{p 2} \cdot B_{p 3}\right)$.

Note that due to the symmetry or the equal tether configuration". the model decouples into three basic subsystems to be discussed subsequentiy. It should also be noted that the components of $x_{p}$ represent small perturbations from nominal trim values: i.e. the values when the tethers are vertical and the spreader bar horizontal. 


\subsection{Three Basic Subsustems and Output Selection}

The three basic subsystems of the TLHS are now discussed, and variables to be commanded by the pilot (outputs) are selected.

The pair $\left(A_{p}, B_{p 1}\right)$ describes the system's "average vertical motion (AVM). This motion involves the $\Sigma \dot{z}$ degree of freedom, and is controlled by issuing average collective commands $\left(\Sigma \theta_{C}\right)$. The subsystem

thus has one input which can be used to control at most ane output, $\Sigma \dot{z}$. This SISO first order subsystem is stable and characterizes the natural damping that occurs during vertical climbs.

The pair $\left(A p_{2}, B p_{2}\right)$ describes the system's "symmetric motion" (SM) [1]. This motion involves the $\Delta x$ and $\Delta \theta$ degrees of freedom, and is controlled by issuing differential cyclic commands $(\Delta \beta \mid c)$. The subsystem thus has only one input which can be used to control at most one output. The variable $\Delta x$ is selected as the output because maintaining horizontal separation is citical. This SISO fourth order subsystem is unstable and is characteristic of any helicopter tethered to a fixed point in space. The instability is referred to as the tethered helicopter mode" (Fig. 3). This mode is a result of the tethers being attached a distance $h$ below the helicopter c.g.'s (Fig. 2) [2].

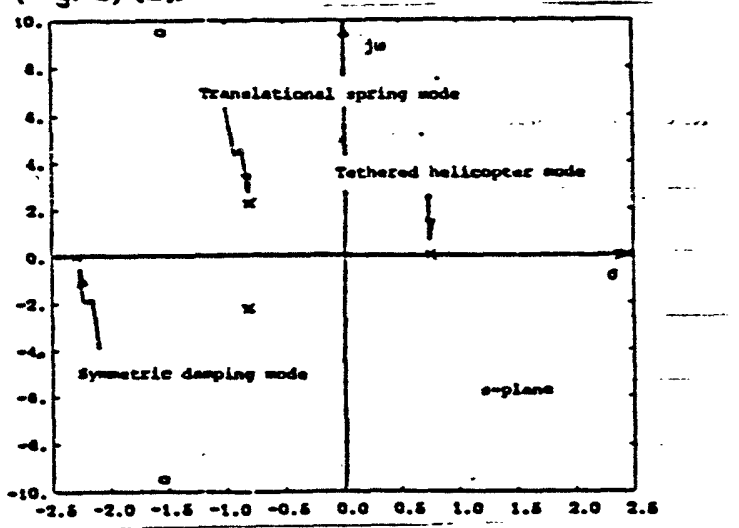

Fig. 3: Symmetric Motion Foles \& Zeros.

Finally. the pair $\left(A_{p 3}, B_{p 3}\right)$ describes the system's anti-symmetric motion" (ASM). This motion involves the $\Sigma \dot{x}, \Sigma \theta, \Delta z, \sum \xi$ degrees of freedom, and is controlled by issuing differential collective commands $\left(\Delta \Theta_{C}\right)$ and average cyclic commands $\left(\Sigma \beta_{l c}\right)$. The subsystem thus has two inputs which can be used to control at most two outputs. The variables $x_{L}-\Sigma x$ and $\Sigma \dot{x}$ are selected as the outputs because they are fundamentally related to the problem of controlling master/slave workload during horizontal flight. The quantity $x_{L}-\Sigma x$ represents the load deviation from the center and is given by: $x_{L}-\Sigma x=h \Sigma \theta+(Z / L) \Delta z+H \Sigma E$

where $h, Z, L$, and $H$ are constant geometric parameters (Fig. 2). This two-input two-output (TITO) seventh order subsystem is unstable, and if $Z=0$, is characteristic of any single hovering helicopter carrying a sling load. The instability here is referred to as the "backflapping mode" (Fig. 4) since it is due to the backflapping of the helicopter main rotors with forward motion [3].

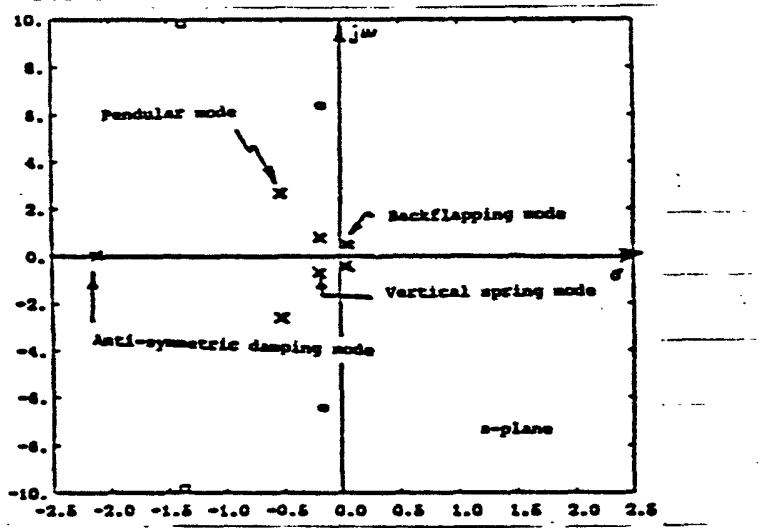

Fig. 4: Anti-Symmetric Motion Poles \& Zeros.

The outputs described above are written in matrix form as follows:

$$
y_{p} \equiv C_{p} x_{p} ; \quad y_{p} \in R^{4}
$$

where

$$
\begin{aligned}
& y_{p}=\left[\Sigma \dot{z}\|x\| x_{L}-\Sigma x \Sigma \dot{x}\right]^{\top} \\
& c_{p}=\operatorname{diag}\left(C_{p 1}, c_{p 2} \cdot C_{p 3}\right)
\end{aligned}
$$

and $C_{p 1}, C_{p 2}, C_{p 3}$ are associated with the AVM, SM, and ASM subsystems, respectively.

Given the above state space model ( $\left.A_{p}, B_{p}, C_{p}\right)$, the TLHS (plant) transfer function matrix is then derined as follows:

$$
\begin{aligned}
G_{p} & \equiv C_{p}\left(s 1-A_{p}\right)^{-1} B_{p} \\
& =\operatorname{diag}\left(G_{p 1}(s), G_{p 2}(s) . G_{p 3}(s)\right)
\end{aligned}
$$

where the $\left\{G_{p i}(s), i=1,2,3\right\}$ specify the input/output characteristics of the three subsystems discussed above, and are given by:

$$
G_{p i}=C_{p i}\left(s l-A_{p i}\right)^{-1} B_{p i} \quad i=1,2,3 .
$$

It is emphasized at this point that the above decoupling into two SISO systems and one TITO system occurs because the helicopters are assumed to be identical and the tethers are assumed to have 
the same length. The "unequal tether oroblem", in which the SM and ASM couple to form a three-input three-output subsystem, is addressed in [4].

It is strongly emphasized that although the equations are decoupled in terms of the defined variables. they remain coupled in certain quantities such as the individual helicopter collective and cyclic controls. Consequently all three subsystems and their corresponding compensators must be evaluated simultaneously so that important quantities can be "untangled" using eqs. (1) - (10). This coupling. in effect, makes controlling the longitudinal twin lift "equal tether configuration" a four-input four-output design problem.

\section{AFCS Structure and Design Specifications}

\subsection{AFCS Structure and Function Definitions}

The final control system structure is shown in Fig. 5 . In this Mimo negative feedback structure there is the plant $G_{p}(s)$ (TLHS). a dynamic augmentation consisting of four integrators (one per channel), and a dynamic $16^{\text {th }}$ order compensator $K_{L Q G / L T R}(\mathrm{~s})$.

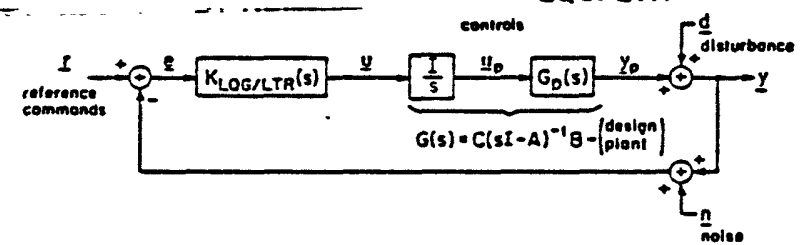

Fig. 5: Structure of AFCS.

The compensator is designed so that the closed loop system meets the specifications presented below. since there are three subsystems, the LQG/LTR compensator has the form:

$$
K_{\text {LQG/LTR }}(s)=\operatorname{diag}\left(K_{1}(s), K_{2}(s), K_{3}(s)\right)
$$

where the $K_{i}(s)$ denote the individual subsystem compensators.

The TLHS (plant) plus the integrators is referred to as the desion olant. Its transfer function matrix is defined as follows:

$$
\begin{aligned}
G(s) & \equiv C(s l-A)^{-1} B \\
& =\operatorname{diag}\left(G_{1}(s), G_{2}(s), G_{3}(s)\right) \\
& =G_{p}(s) / s
\end{aligned}
$$

where $A \equiv \operatorname{diag}\left(A_{1}, A_{2}, A_{3}\right)$

$$
\begin{aligned}
& B=\operatorname{diag}\left(B_{1}, B_{2}, B_{3}\right) \\
& C \equiv \operatorname{diag}\left(C_{1}, C_{2}, C_{3}\right)
\end{aligned}
$$

and the $i^{\text {th }}$ design plant input/output characteristic is given by:

$$
\begin{aligned}
G_{i}(s) & \equiv C_{i}\left(s l-A_{i}\right)^{-1} B_{i} \\
& =G_{p i}(s) / s \quad i=1,2,3
\end{aligned}
$$

where $i=1,2,3$ denote the AVM, SM, and ASM subsystems respectively.

In an analogous manner. the final loop. sensitivity. and closed loop transfer function matrices can be def ined as follows:

$$
\begin{aligned}
& G_{L}(s) \equiv \operatorname{diag}\left(G_{L 1}(s), G_{L 2}(s), G_{L 3}(s)\right) \\
& S_{F}(s) \equiv \operatorname{diag}\left(S_{F 1}(s), S_{F 2}(s), S_{F 3}(s)\right) \\
& T_{F}(s) \equiv \operatorname{diag}\left(T_{F 1}(s), T_{F 2}(s), T_{F 3}(s)\right) .
\end{aligned}
$$

3.2 Performance Specifications -

To develop reasonable performance specifications. i.e. specifications which are "passenger friendly", the piant singular values (Fig. 6) were analyzed and closed loop transient responses were studied to get a feel for typical twin lift pilot maneuvers.

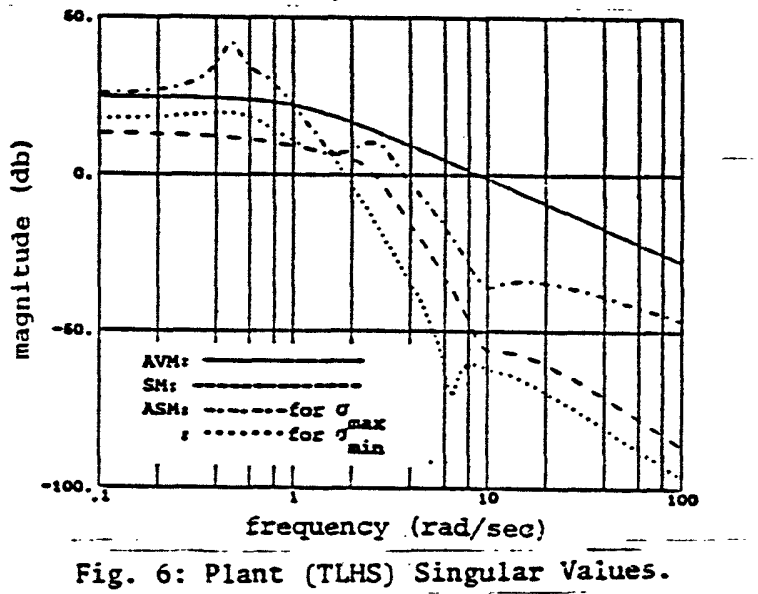

One performance specification is zero steady state error to pilot step reference commands, $I$ for $\Sigma \dot{z}, \Delta x$, $x_{L}-\Sigma x$, and $\Sigma \dot{x}$. Another is complete rejection of step disturbances, $d$ with. respect to the above four output variables. The four integrators introduced in the AFCS (one per channel) guarantee that both of these performance specifications are met (see Internal Model Principle (71). The integrators also help with low frequency command following. low frequency disturbance rejection, and low frequency sensitivity. To guarantee that these properties are built into the design, it is required that the final subsystem sensitivity functions satisfy the following frequency domain constraints:

$\left|S_{F}(j \omega)\right|<-20$ db for all $\omega<.08 \mathrm{rad} / \mathrm{sec}$

$\left|S_{F 2}(j \omega)\right|<-20$ db for all $\omega<.04 \mathrm{rad} / \mathrm{sec}$

$\sigma_{\text {max }}\left[S_{F j}(j \omega)\right]<-20 \mathrm{db}$ for all $\omega<.06 \mathrm{rad} / \mathrm{sec}$ (37) 
where $\sigma_{\max }[\cdot]$ denotes the maximum singular value.

\subsection{Robustness Specifications}

To be robust with respect to the high frequency unmodeled main rotor dynamics (at $\omega \approx 27 \mathrm{rad} / \mathrm{sec}$ ). the final subsystem closed loops must satisfy the following frequency domain constraints:

$\left|T_{F i}(j \omega)\right|<-20$ db for all $\omega>10 \mathrm{rad} / \mathrm{sec}$

$\left|T_{F 2}(j \omega)\right|<-40$ db for all $\omega>10 \mathrm{rad} / \mathrm{sec}$

$\sigma_{\max }\left[T_{F 3}(j \omega)\right]<-20 \mathrm{db}$ for all $\omega>10 \mathrm{rad} / \mathrm{sec}$

These also help in attenuating high frequency sensor noise, $\underline{\text {. }}$

The above performance and stability robustness specifications are nonsensical uniess the nominal closed loop system is stable. This , however. is not a problem since the LQG/LTR design methodology will be used. The methodology guarantees nominal stability and offers excellent stability margins [7].

\section{LQG/LTR Design Methodology}

\subsection{Obtaining the design}

Next the LQG/LTR design methodology is applied to the minimum phase [6] [9] [10] design plant $G(s)=$ $C(s:-A)^{-1}$ (S (i.e., TL4S plus integrators).-Fine design process consists of two steps:

Step 1: Desigri a "nice target loop", $G_{K F}(s) \equiv C(s l-A) H$, where $H$ is found by solving a filter algebraic Riccati equation The Riccati equation depends on design parameters that can be manipulated to place loop transmission zeros at appropriate locations to give us loop shapes that satisfy the aforementioned "passenger iriendly" specifications. $G_{K F}(s)$ has guaranteed closed loop rabustness properties.

Step 2: "Recover" the nice loop properties of $G_{K F}(s)$

from the design plant:

LTR: $G(s) K_{L Q G / L T R}(s) \longrightarrow G_{K F}(s)$

$$
\rho_{1} \cdot \rho_{2} \cdot \rho_{3} \stackrel{\text { as }}{\longrightarrow} 0 \text {; }
$$

i.e. as the design parameters $p_{1} \cdot p_{2} \cdot p_{3}$ (one for each subsystem) get small the loop transfer function matrix approaches a function with good performance and guaranteed robustness properties.

4.2 Evaluation of the final design

The final loop. sensitivity, and closed loop singular values appear in Figs. $7 a-7 c$. The shaded regions indicate that the design specifications (eqs. $(37)-(40))$ have been met.

Fig. 7a shows that all loop singular values have gain-crossover frequencies less than $1.5 \mathrm{rad} / \mathrm{sec}$ and above $.6 \mathrm{rad} / \mathrm{sec}$. Their low frequency slopes of -20 $\mathrm{db} / \mathrm{dec}$ confirms the presence of the four integrators in the loop.

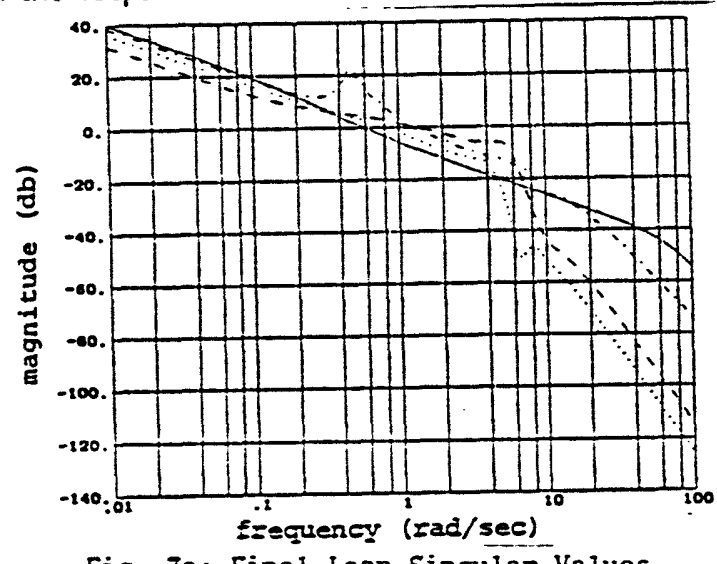

Fig. 7a: Final Loop Singular Values.

Fig. $7 \mathrm{~b}$ shows that all sensitivity singular values lie below the -20 do "performance region" thus guaranteeing good low frequency command following, good low frequency disturbance rejection, and good low frequency sensitivity reduction. The poor SM and ASM sensitivity properties at irequencies above .6 $\mathrm{rad} / \mathrm{sec}$ are expected since these subsystems have unstable modes which force the tradeoff of nice feedback properties at high frequencies for nice feerback properties at low frequencies [9] [10].

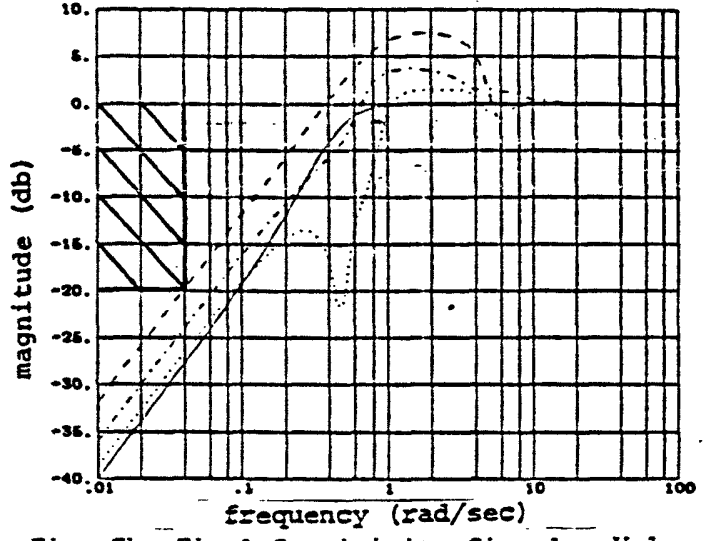

Fig. 7b: Final Sensitivity Singular Values.

Fig. 7c shows that all closed loop singular values lie below the -20 do "robustness region" thus guaranteeing robustness to the unmodeled rotor dynamics at $\omega \approx 27 \mathrm{rad} / \mathrm{sec}$. The design also provides a degree of high frequency sensor noise attenuation built into the design.

To complete the evaluation of the design, the following reference commands were issued, assuming zero initial conditions, no disturbances $(d=\underline{0})$, and no sensor noise $(n=0)$ : 


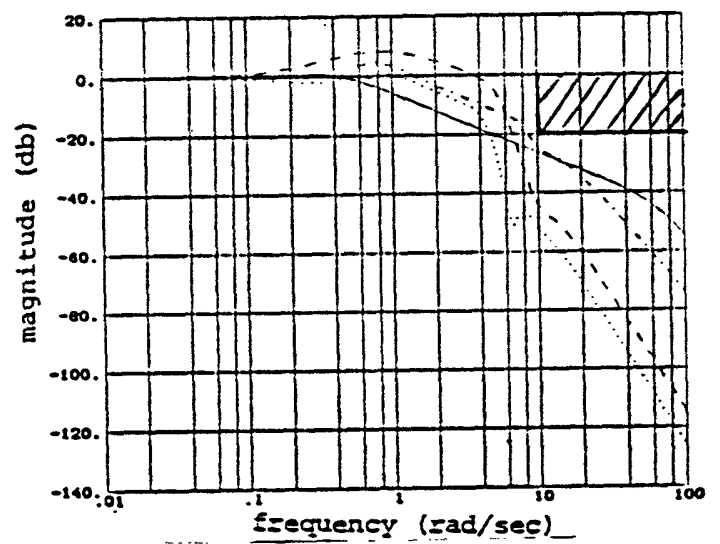

Fig. 7c: Final Closed Loop Singular Values. Reference Command

AVM: $\Sigma \dot{z}=5 \mathrm{rt} / \mathrm{sec}$

SM: $\Delta x=2 \mathrm{rt}$

ASM:

$$
x_{L}-\Sigma x=-1 \mathrm{ft}
$$$$
\Sigma \dot{x}=5 \mathrm{ft} / \mathrm{sec}
$$

The results of the above linear simulation are given in Figs. $8 a-8 c$ and $9 a-9 d$. It is emphasized that eqs. (1) - (10) are used to "untangle" the variables of interest (e.g. the individual helicopter velocities and controls).

Initially the helicopters are at rest with the tethers vertical and the spreader bar horizontal. The pilot reference commands being issued ask for an effective system steady state motion of $7.071 \mathrm{ft} / \mathrm{sec}$ in a direction 45 degrees with respect to the horizontal, with an incremental horizontal separation of 2 feet $(\Delta x=2)$. and the payload shifted 1 foot to the iefit of the center $\left(x_{1}-\Sigma x=-1\right)$.

Figures $8 \mathrm{a}$ and $9 \mathrm{a}$ show how the AFCS coordinates the helicopter controls so as to achieve the outputs commanded by the pilot The initial control transients, altnough tolerable, are relatively large but this is expected since the AFCS is trying to achieve a vertical climb of $5 \mathrm{ft} / \mathrm{sec}$, a horizontal drive of 5 $\mathrm{ft} / \mathrm{sec}$, keep the load $1 \mathrm{ft}$ behind the center, all while maintaining an incremental horizontal separation of 2 feet between two inherently unstable 14000 ib Blackhawks!

Fig. 8 a shows that the AFCS keeps the master and slave collectives out of phase throughout the 10 second maneuver. Fig. 80 shows the same for the helicopter velocities.

The net efrect is seen in Fig. $8 \mathrm{c}$ which shows how the AFCS effectively uses the $\Delta z$ degree of freedom to control the $x_{L}-\sum x$ load motion After approximately 6 seconds, the $\Delta z$ and $x_{L}-\sum x$ motions come into phase

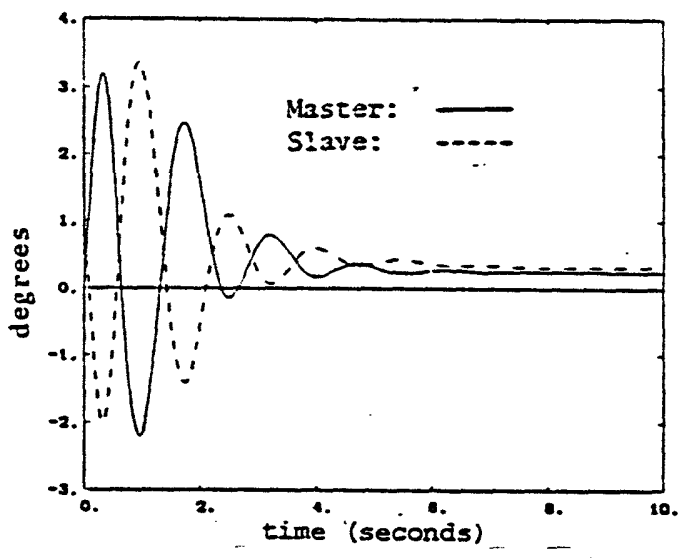

Fig. 8a: Master \& Slave Collective Controls.

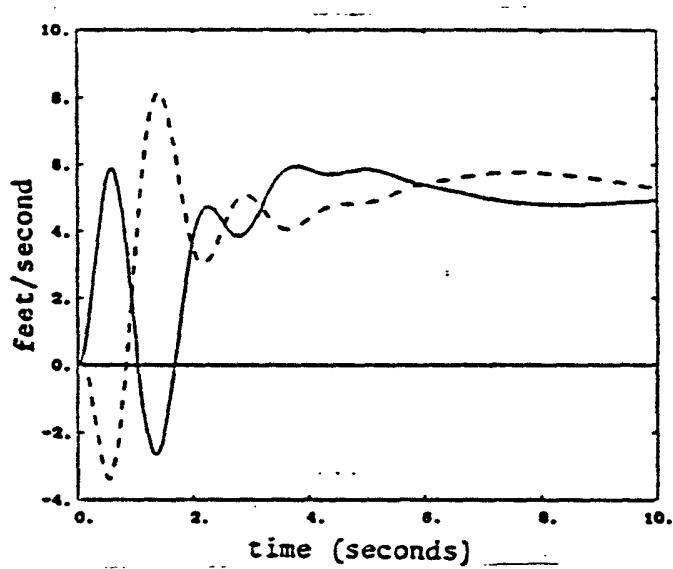

Fig. 8b: Master \& Slave Vertical Velocities.

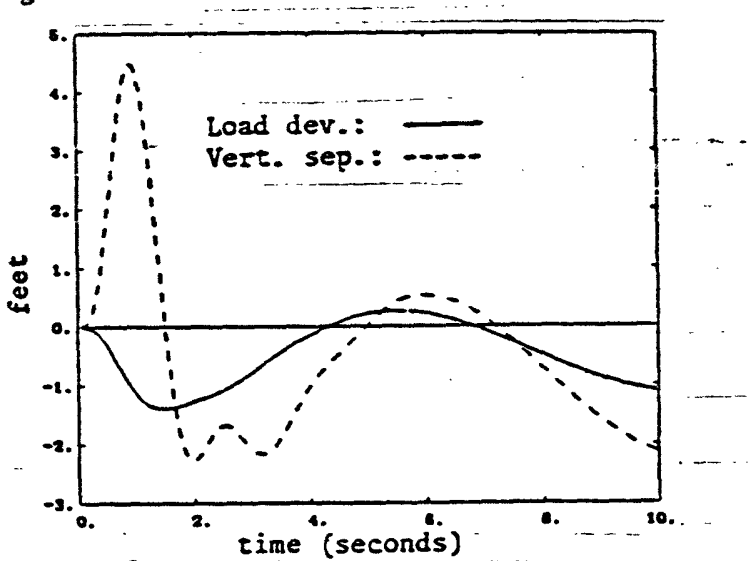

Fig: 8c: Load Dev. from Center \& Vert. Sep.

with one another and decay to their correct steady state values of -2 feet and -1 foot, respectively. The motion observed is essentially the vertical spring mode associated with the anti-symmetric motion (Fig. 4). in the steady state a difference in collective controls provides the force to maintain the slave above the master $(\Delta z=-2)$. This in turn maintains the payload to the left of the center $\left(x_{L}-\Sigma x=-1\right)$. The key point to be made here is that the AFCS must 
"tradeoff" the system $\Delta z$ motion in order to follow pilot reference commands in $x_{L}-\Sigma x$. It thus follows intuitively that relatively poor vertical acceleration characteristics may result as a consequence of requiring the use of vertical separation to control the load deviation from the center. This is somewhat apparent in figure $8 \mathrm{~b}$ which exhibits the expected vertical acceleration characteristics. It must be pointed out that the vertical acceleration characteristics can be improved by increasing the bandwidth of the final AVM and ASM loop, but oniy at the expense of larger collective controls, and the possible excitation of the high frequency unmodeled rotor dynamics.

Fig. 9a shows that the AFCS initially maintains the helicopter cyclics slightly out of phase. In doing so both helicopters pitch downward (Fig. 9b) and accelerate forward (Fig. 9c). In trying to achieve a 2 foot horizontal separation, the AFCS coordinates the cyclics so as to induce the necessary helicopoter pitching motions (Fig. 9c). Due to the inertia of the helicopters, however, a horizontal separation overshoot" occurs (Fig. 90). This overshoot can be decreased substantially by increasing the bandwidth of the final SM but this can be done only at the expense of substantially larger cyclic controls and the possible excitation of the high frequency unmodeled rotor Gynamics.

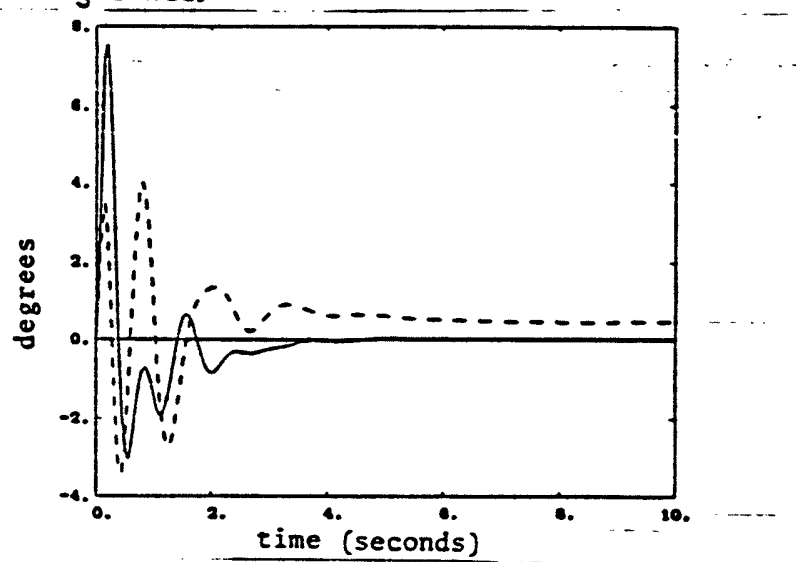

Fig. 9a: Master \& Slave Cyclic Controls.

Figs. 9a - 9d show that the AFCS must essentially "tradeoff" the pitching motions of the helicopters in order to follow pilot reference commands for $\Delta x$. Because of this tradeoff, it follows that a sluggish horizontal acceleration characterictic may result for a low bandwidth SM design (Fig. 9c). As in the vertical case discussed above, it should be noted that the

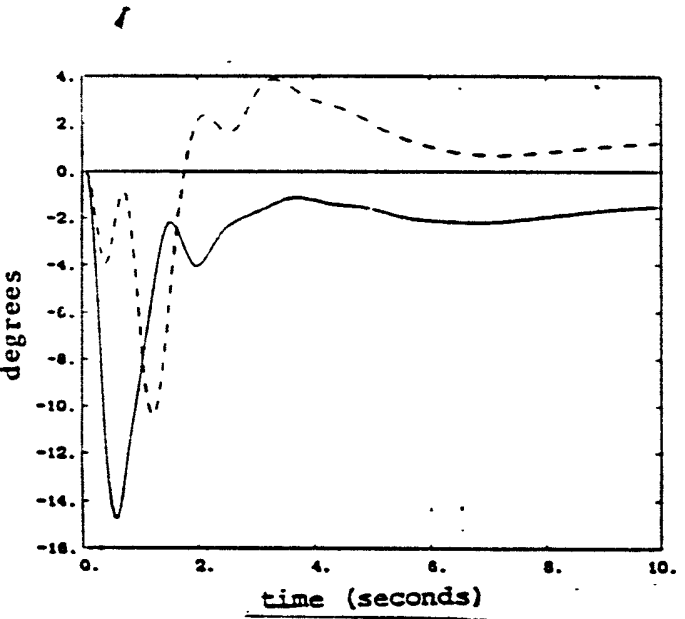

Fig. 9b: Master \& Slave Pitch Attitudes.

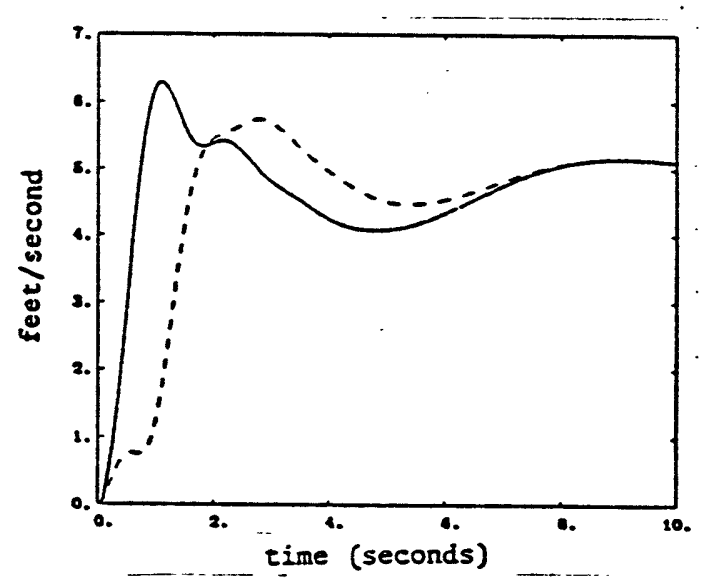

Fig. 9c: Master \& Slave Horizontal Velocities.

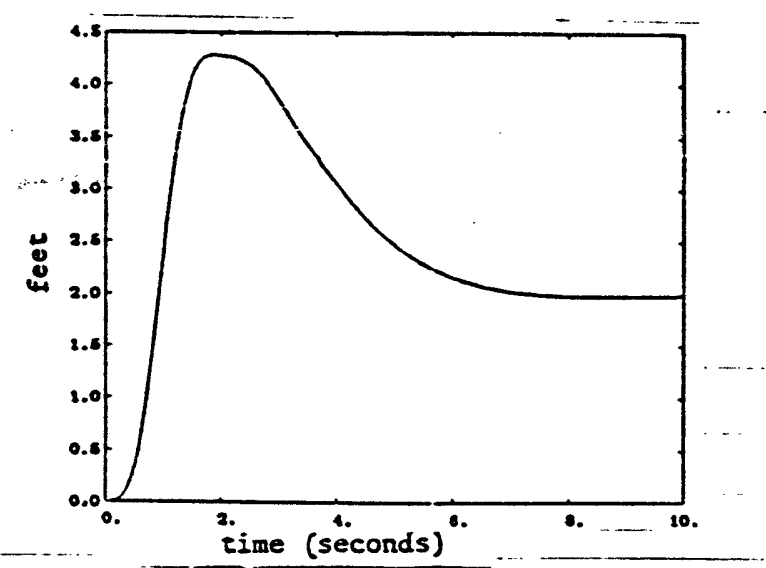

Fig. 9d: Horizontal Separation.

horizontal acceleration charateristics may be greatly improved by increasing the bandwidth of the SM loop. but only at the expense of larger cyclic controls, larger pitch rates, and the possible excitation of the high frequency unmodeled main rotor dynamics.

Comparing figures $8 \mathrm{~b} \& 9 \mathrm{c}$, it is seen that the vertical acceleration charateristics are slightly worse than the horizontal acceleration charateristics. This is 
primarily due to the fact that although $\Delta z$ does appear in the expression for $x_{L}-\sum x$ (eq. 16), it is not directly "penalized"; i.e. it is not an output as is its horizontal counterpart, $\Delta x$.

It should be noted that the four integrators (one per input channel) have provided the desired zero steady state error to the pilot's step reference commands. It should also be noted that because of the unstable "tethered helicopter" and "backflapping" modes, a minimum bandwidth is required to just stabilize the symmetric and anti-symmetric motions. This, of course. translates into minimum control rates and more importantly (from a passenger's viewpoint). larger pitch rates. These large pitch rates must be lived with, and are linear functions of the pilot reference commands as well as the system initial conditions.

To improve the vertical performance one might try first to use a configuration in which the slave tether is longer than the master tether [4]. Such a configuration greatly improves the relative stability of the nominal model by introducing damping in the "tethered helicopter" mode and the "backflapping" mode, as well as the other oscillatory modes of the TLHS. It must be emphasized that by bringing the unstable poles of the TLHS closer to the left half plane, the minimum bandwidth needed to stabilize our system decreases. This is desirable because a lower bandwidth implies smaller controls and hence smaller pitich rates.

Another great improvement would result by fiying with the helicopter-tether attachment points directly at the Blackhawk c.g.'s $(h=0)$. This has been proposed at Sikorsky Aircraft and is dicussed in [I]

Another idea for improving the performance of the TLHS is to use real time tether length control. This would introduce another degree of freedom in the controls which would seem to be of tremendous help in controlling the payload-bar assembly.

\section{Summary}

This paper has shown that the LQG/LTR design methodology, coupled with singular value ideas, can be used to formulate performance and stability robustness specifications which can then be used to develop a centralized MIMO AFCS for a twin lift hehicopter system (TLHS). The methodology is extremely powerful in that it possesses enough degrees of freedom to tailor designs to prescibed pilot specifications. The paper and [4] show that the methodology can be used to address the TLHS control problem with repect to the following five fundamental reedback issues:

(1) low frequency command following.

(2) low frequency disturbance rejection,

(3) low frequency sensitivity reduction,

(4) robustness to high frequency modeling, and

(5) attenuation of high frequency sensor noise.

\section{References}

[11. H.C. Curtiss and F.W. Warburton -Stability and Control of the Twin LIft Helicopter System: presented at 40 th Annual forum of Arlington, Va., May 16-18, 1984.

[2]. $L$ Kaufman and ER. Schultz, The Stability and Control of Tethered Helicopters", JAHS Vol. 7, No. 4, October 1962

[3l. AR.S. Bramwell, Helicopter Dunamics, John Wiley and Sons. New York, 1976.

[4L A.A. Rodriguez, Multivariable Control of a Twin Lift Helicopter System using the LQG/LTR Design Methodology", SM Thesis, MIT, May 1986.

151. J.C. Doyle, and G. Stein Multivariable Feedback Design: Concepts for a Classical Modern Synthesis", IEEE Trans. on Auto. Control Vol. AC-26, No. 1, DP. 4-16.

[6]. G. Stein and $M$ Athans, The $L Q G / L T R$ Procedure for Multivariable Feedback Control Design:, Tech. Paper. MIT LIDS-P-1304, May i984.

171. $M$ Athans, 5.232 class notes, MIT, Spring 1985.

[81. N.A. Lehtomaki, NR. Sandell, and M. Athans, Robustness Results in LQG Based Multivariable Control Designs". IEEE Trans. on Auto. Control Vol. AC-26, No. 1, pp. 75-93, Feb. 1981.

[9L. J.S. Freudenberg, and D.P. Looze. "The Impact Right Half Plane Poles and Zeros and Design Trade-offs in Feedback Systems", Tech Report, Coordinated Sci. Lab, Univ. Illinois, 1983.

[10!. S. Boyd, and C.A. Desoer, "Subharmonic Functions and Performance Bounds on LTI Feedback Systems". U.C. Berkeley. Memorandum No. UCB/ERL M84/5I, II June 1984. 